\title{
Identification of a canonical configuration for a quantitative analysis of transport phenomena in electric machines based on entropy generation
}

\author{
T. Eger ${ }^{1,2}$, D. Thévenin ${ }^{1}$, G. Janiga ${ }^{1}$, T. Bol ${ }^{2} \&$ R. Schroth ${ }^{2}$ \\ ${ }^{1}$ Laboratory of Fluid Dynamics and Technical Flows, \\ University of Magdeburg "Otto von Guericke”, Germany \\ ${ }^{2}$ Robert Bosch GmbH, Germany
}

\begin{abstract}
The demand for higher power density at low costs leads to an increasing importance of thermal management in electrical machines. Different approaches are employed to develop cooling strategies. However, a truly quantitative analysis is barely used, partly due to the huge data quantity generated by the flow simulation. In many cases only visual postprocessing under consideration of a few variables is implemented. The present paper provides an overview about a method suitable for investigating transport phenomena induced by forced convection. Different configurations are retained to check the generality of the approach. Based on the second law of thermodynamics, the entropy generation in the fluid field is considered. As an example of external flow, a semi-infinite plate is examined. For internal flows, two rotating, concentric cylinders are then analysed. Finally, transport processes in the flow between these cylinders located in a rectangular domain $(\Omega)$ are analyzed. In addition, the outer cylinder has two openings along the main stream direction. The straight flow imposed at the inlet velocity of the domain $(\Omega)$ is influenced by the rotational motion of the inner cylinder as well as by the opening angle $(\beta)$ at the outer cylinder and the radius ratio between the two cylinders $(I)$. The thermal boundary conditions are isothermal or isoflux and isothermal or adiabatic for the inner and outer cylinder, respectively. In a first step, numerical calculations of the Nusselt number $(\mathrm{Nu})$
\end{abstract}


are discussed. This methodical approach can be a first step to analysing transport phenomena under consideration of physically-based indicators.

Keywords: transport phenomena, entropy generation, rotating cylinder, heat transfer, cooling, electrical machines.

\section{Introduction}

Cooling by forced convection has a wide range of application in engineering. To increase the lifetime of electric devices, the objective is to maintain the operating temperature at a safe level. Inside an alternator, fan blades generate a pressure gradient, due to the rotational motion. Air from the engine bay streams through the alternator and thereby increases the enthalpy transport. With the help of computational fluid dynamics (CFD) the flow field can be analyzed. The main objective is a better design while considering certain constraints.

In [1] Thévenin and Janiga showed how to combine Evolutionary Algorithms with CFD (an approach also called CFD-O). In chemical engineering the Nusselt number $(\mathrm{Nu})$ can be used as an efficiency objective to design different kinds of system configurations as presented by the VDI Heat Atlas [2]. The fundamental work of Bejan [3] introduced a new way to analyse different processes based on the second law of thermodynamics. The consideration of exergy destruction due to conduction and fluid friction offers a new way to look at heat transfer problems. Moreover, the entropy generation is a postprocessing value provided by a volume integral over the domain of interest. Fowler and Bejan [4] showed optimal sizes of bodies for external flows with the objective of entropy generation minimization (EGM). Herwig and Schmandt [5] used the second law analysis (SLA) to determine the drag coefficient for external flows with the help of the volumetric entropy generation in the flow field. In contrast to external flows, SLA-approaches for internal flows are widely available. Wenterodt and Herwig [6] and Herwig et al. [7] showed that an optimal Reynolds number $(R e)$ exists for a heated pipe considering EGM. Ko and Tink [8] and Ko [9] investigated EGM for rectangular duct geometries with longitudinal ribs. Şahin [10] varied duct geometries, gave an analytical formulation and estimated the best geometry based on EGM. Aziz [11] summarized analytical studies of entropy generation in Couette flow for different kinds of boundary conditions. Mahmud and Fraser [14] and Mahian et al. [17] presented an analytical solution for the entropy generation between two concentric rotating cylinders. Mirzazadeh et al. [13] extended the analytical solution for non-linear viscoelastic fluids. Yilbas [18] computed temperature rise and entropy generation due to conduction and fluid friction.

Based on the same geometry of two rotating, concentric cylinders, the present authors included openings along the outer cylinder. Hence, it is more accurate to speak of an outer sleeve instead of a cylinder for this case. The idea is to involve different types of transport phenomena for testing physically-based indicators, e.g. entropy generation. For this purpose a configuration is needed, which is able to involve a variety of flow fields dominated by different heat transport mechanism. 


\section{Physical model}

Based on conceptual considerations in the fields of aerodynamic and transport phenomena, the authors developed a canonical configuration that will be systematically used for further analysis.

\subsection{Conceptual considerations}

Figure 1 shows two fundamental cases for aerodynamic studies. Because of the employed boundary conditions, velocity $\delta$ and thermal $\delta_{T}$ boundary layers develop along the bodies.

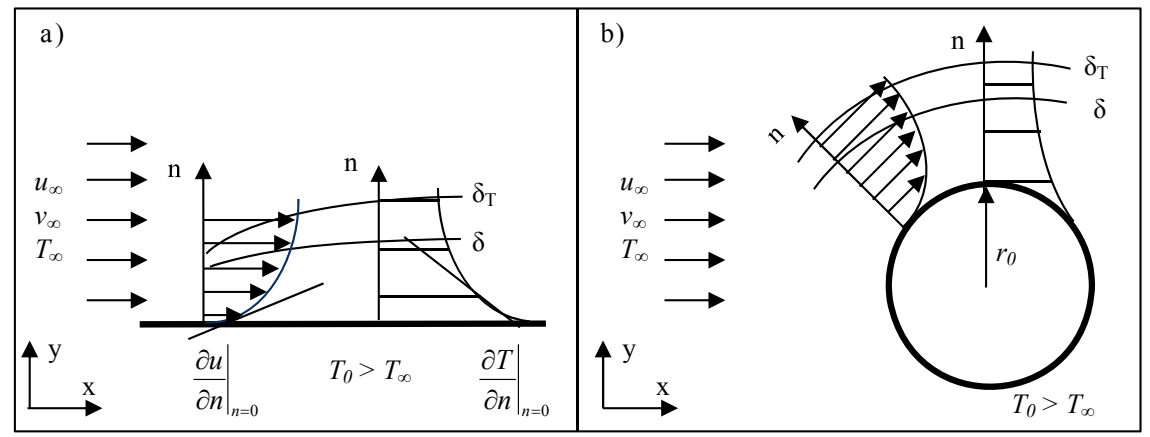

Figure 1: Boundary layer formation for a) semi-infinite flat plate and b) cylinder.

Near the wall, velocity and temperature gradients are high. A high Nusselt number results in an increasing velocity gradient. For the semi-infinite flat plate in fig. 1a), the Reynolds Analogy can be used to relate the flow parameters to the thermal parameters. In this case, $N u$ can be formulated as:

$$
N u=\frac{h L}{k}=\frac{c_{f}}{2} R e
$$

where $h$ is the local heat transfer coefficient, $L$ is the characteristic length and $k$ is the thermal conductivity of the fluid. $R e$ is the Reynolds number and $c_{f}$ is the skin friction coefficient, defined as:

$$
\begin{gathered}
R e=\frac{\rho u_{\infty} L}{\mu} \\
c_{f}=\frac{2 \tau}{u_{\infty}^{2} \rho}=\left.\frac{2 \mu}{u_{\infty}^{2} \rho} \frac{\partial u}{\partial n}\right|_{n=0} .
\end{gathered}
$$

Thereby $\rho$ defines the density of the fluid, $\mu$ the dynamic viscosity, $u_{\infty}$ the ambient velocity and $\tau$ the shear stress. In such cases, the velocity gradient could 
be a criterion to characterize heat and mass transfer. The transport of energy in form of heat is often described by the kinetic approach:

$$
Q=h A\left(T_{w^{-}} T_{\infty}\right)
$$

From eqs. (1) and (4) it is obvious that the transport is related to the gradients near the wall and the temperature difference $\Delta T=T_{0}-T_{\infty}$. This results in two different kinds of processes:

- near-wall processes (local) and

- enthalpy transport on a global scale.

Extending now the geometry from fig. 1b) with a vertical plate on the left hand side, the flow profile will change like described in fig. 2.

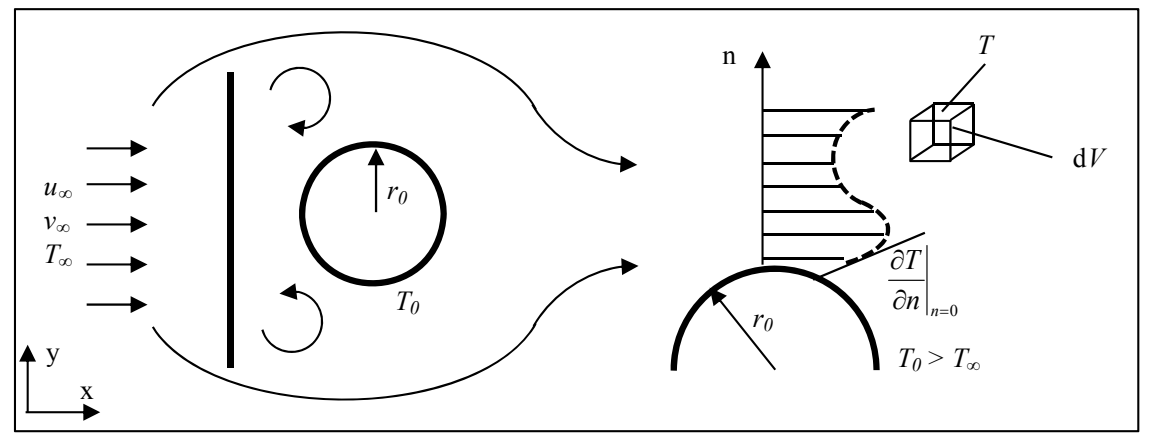

Figure 2: Temperature boundary layer profile for a specific case with interaction.

In comparison to the cases in fig. 1, a reference temperature $T$ in fig. 2 is difficult to determine due to complex processes in the flow field. Based on the first law of thermodynamics, the change of enthalpy due to heat transfer can be calculated with:

$$
d \dot{q}=d \dot{h}=c_{p} \Delta T
$$

where $h$ is the specific enthalpy and $c_{p}$ the specific heat capacity for constant pressure. This small change in the model shows the difficulty of finding a useful reference temperature $T$. Fig. 3 illustrates another variation starting from a basic case of aerodynamics. In fig. 3a) the boundary layer developing along a flat wall interacts with a rotating cylinder placed inside a wall recess. When increasing the angular velocity $\omega_{0}$ of the cylinder, energy transfer in form of heat is improved (see eqn. (1)). In fig. 3b) the same structure is used but with a much deeper cavity $\left(l_{2}>>l_{1}\right)$. In this case, the geometric interaction with the rotating cylinder is different. Assuming that the angular velocity of the cylinder is the same in both cases, the velocity gradient will be the same, too. Using the Reynolds analogy, no deviation between both Nusselt numbers is identifiable. However, in fig. 3b) less heat and mass transfer obviously occurs, due to the larger value of $l_{2}$. 


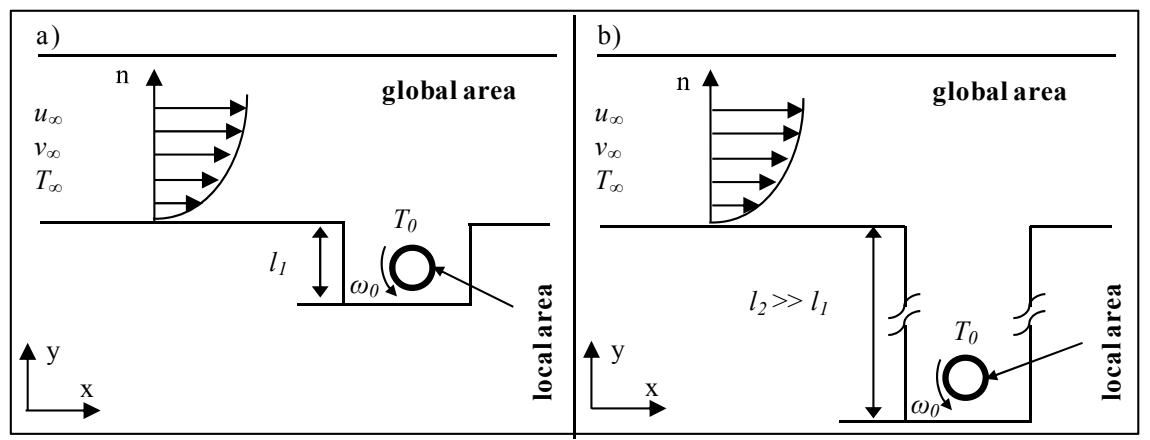

Figure 3: Influence of a design parameter on heat and mass transfer.

\subsection{Description of the canonical configuration}

To investigate transport phenomena and to distinguish between local and global processes, the authors propose now a new numeric configuration.

It is physically based on the cases described above. The objective is to describe a variety of relevant processes with the same geometrical configuration and involving only a small number of parameters. The retained geometry is shown in fig. 4 and involves a rotating cylinder with a surrounding sleeve that has two openings along the main flow direction.

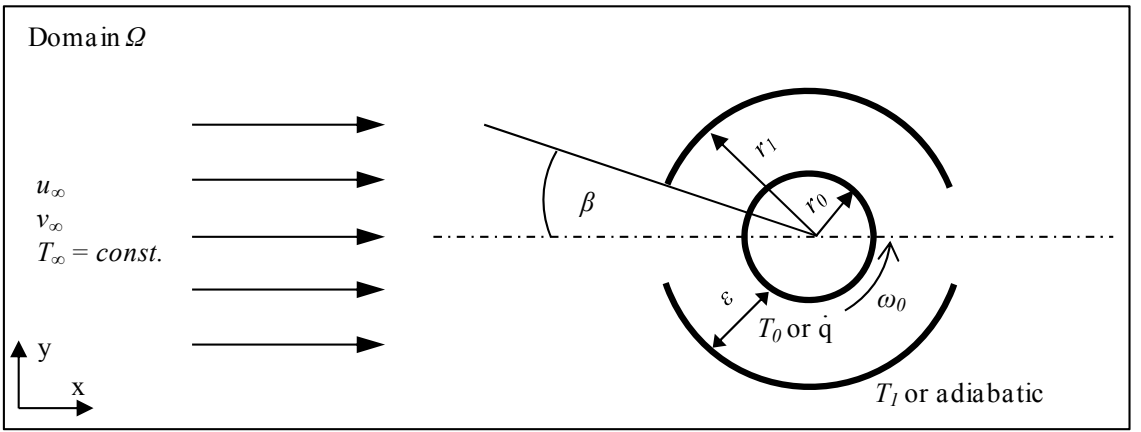

Figure 4: Canonical configuration: Geometry of the rotating cylinder with open sleeve in computational domain $\Omega$.

The opening angle $\beta$ defines the openings on both sides, equally. Its range is obviously defined with $0 \leq \beta \leq \pi / 2$. The gap $\varepsilon$ between the cylinder and the external sleeve is strictly positive. The flow is strongly influenced by the surrounding sleeve. Compared to the isolated rotating cylinder, two additional design parameters have been added $(\beta$ and $\varepsilon)$. Table 1 gives an overview of the parameters which have an influence on the flow field locally (within the annuli) as well as globally (in the whole domain $\Omega$ ). 
Table 1: $\quad$ Parameters influencing the flow field.

\begin{tabular}{|l|c|c|}
\hline & locally & globally \\
\hline dynamic & $u_{0}$ & $u_{\infty}$ \\
\hline heat transfer & $\Delta T, u_{0}$ & $\Delta T, u_{\infty}$ \\
\hline design & $\varepsilon$ & $\beta$ \\
\hline
\end{tabular}

The ratio of inner $\left(r_{0}\right)$ and outer radius $\left(r_{l}\right)$ is defined as $\Pi$. Due to that the gap $\varepsilon$ can be computed as $(\Pi-1) r_{l}$. The dimensionless temperature is $\Phi=\left(T-T_{0}\right) / \Delta T$ with $\Delta T=T_{1}-T_{0}$ (assuming that $T_{0}<T_{1}$ ). The dimensionless temperature difference is $\Lambda=\Delta T / T_{0}$. The Brinkman number $(\mathrm{Br})$ is defined as the ratio between the thermal energy which is produced due to dissipation and the ability of the fluid to evacuate this energy:

$$
B r=E c \operatorname{Pr}=\frac{\left(r_{0} \omega_{0}\right)^{2} \mu}{k \Delta T} .
$$

The cylinder and sleeve are mounted in a rectangular domain $\Omega$, which is constructed as three-dimensional with symmetry boundary conditions in the cross directions. The flow is considered as steady and laminar. Ideal gas has been chosen as working fluid with the properties of air. Due to the small temperature change in the flow field, the thermo-physical properties such as dynamic viscosity $(\mu)$ and thermal conductivity $(k)$ are assumed to be constant. Considering the model description, the physical model becomes a single cylinder at the limit case of $\beta=\pi / 2$. Therefore, the characteristic length scale for $R e$ (see eqn. (2)) is defined with $2 r_{0}$ and the characteristic velocity is $u_{\infty}$. Considering eqs. (1)...(3), $N u$ becomes:

$$
N u=\left(2 r_{0}\right)^{2} \frac{\omega_{0}}{2 u_{\infty} \varepsilon} .
$$

Assuming that $\varepsilon<<2 r_{0}$, the velocity profile in the annuli can be considered as linear. When the design parameters $r_{0}$ and $\varepsilon$ are fixed, only the dynamic parameters have an influence on the Nusselt number. Using this canonical configuration it is possible to analyse separately the influence of $u_{0}$ (locally) and $u_{\infty}$ (globally). Regarding eqn. (2) the Reynolds number is defined as $R e=u_{\infty} 2 r_{0} / v_{\infty}$. Based on similarity theory, the angular velocity $\omega_{0}$ can be expressed in a dimensionless form, too.

Table 2: Dimension matrix and relevant quantities.

\begin{tabular}{|l|c|c|c|c|c|c|c|c|c|}
\hline & $h o$ & $\omega_{0}$ & $u_{\infty}$ & $2 r_{0} \sin \beta$ & $\rho$ & $c_{p}$ & $2 r_{0}$ & $k$ & $v_{\infty}$ \\
\hline mass & 1 & 0 & 0 & 0 & 1 & 0 & 0 & 1 & 0 \\
\hline length & 0 & 0 & 1 & 1 & -3 & 2 & 1 & 1 & 2 \\
\hline temp. & -3 & -1 & -1 & 0 & 0 & -2 & 0 & -3 & -1 \\
\hline time & -1 & 0 & 0 & 0 & 0 & -1 & 0 & -1 & 0 \\
\hline
\end{tabular}

Table 2 shows the corresponding dimension matrix with the relevant quantities characterizing the physical model shown in fig. 4. After solving the equations, the dimensionless tangential velocity $(I)$ can be defined as: 


$$
\Gamma=\frac{\omega_{0}\left(2 r_{0}\right)^{2}}{v}
$$

\section{Numerical method}

All the equations are discretised with a finite-volume formulation relying on the Reynolds-Averaged Navier-Stokes (RANS) approach with a second-order upwind scheme. For the mesh, hexahedra elements are chosen. For the calculation of $N u$, a reference temperature $\left(T_{B u l k}\right)$ is needed. As suggested in Zhang et al. [21] $T_{\text {Bulk }}$ was defined with the ambient temperature $T_{\infty}$. The viscous work term was included in the energy equation, since it is needed for calculations with high velocity gradients. The convergence criteria for mass, velocity components and enthalpy is set as a factor of $10^{-6}$. There was no difference in the convergence behaviour with including viscous work term.

\section{Grid-independence test and code validation}

In this section, the dependency of the grid on a semi-infinite flat plate is tested, as well as the implemented numerical code on two concentric, rotating cylinders.

\subsection{External flow: semi-finite plate}

Herwig and Schmandt [5] considered the drag coefficient $\left(c_{D}\right)$ for an infinite plate as follows:

$$
c_{D}=2\left[1.328 R e^{-1 / 2}+2.67 R e^{-7 / 8}\right] .
$$

For an alternative formulation of $c_{D}$, they suggested to use the SLA-approach (entropy generation occurring due to fluid friction only) for the calculation. Based on the Gouy-Stodola-Theorem $c_{D}$ can be expressed as:

$$
c_{D}=\frac{T}{\frac{\rho}{2} u_{\infty}^{3} A} S_{F}^{\prime \prime \prime} .
$$

where $S_{F}^{\prime \prime \prime}$ is the entropy generation due to fluid friction. Fig. 5 compares the results of eqn. (10) with eqn. (9), showing the excellent agreement between both results in the relevant range for $R e$.

A grid-independence test was performed for the semi-infinite plate. For a laminar flow, the results are matching very well with each other, under the condition that $y^{+} \leq 1$. For the first mesh, $y^{+}>1$ is observed for $R e>4096$. Around this Reynolds number the red dotted line starts diverging from the expected solution and leads to increasingly wrong results, strongly underestimating $c_{D}$. The same effect appears for the second mesh around $R e=100000$ (dashed line). The third and finest mesh show a good agreement with the analytical results till $R e>500,000$, since $y^{+}<1$ always apply. These results corroborate the recommendations of ANSYS [22], since $y^{+}<1$ is recommended for high accuracy. 


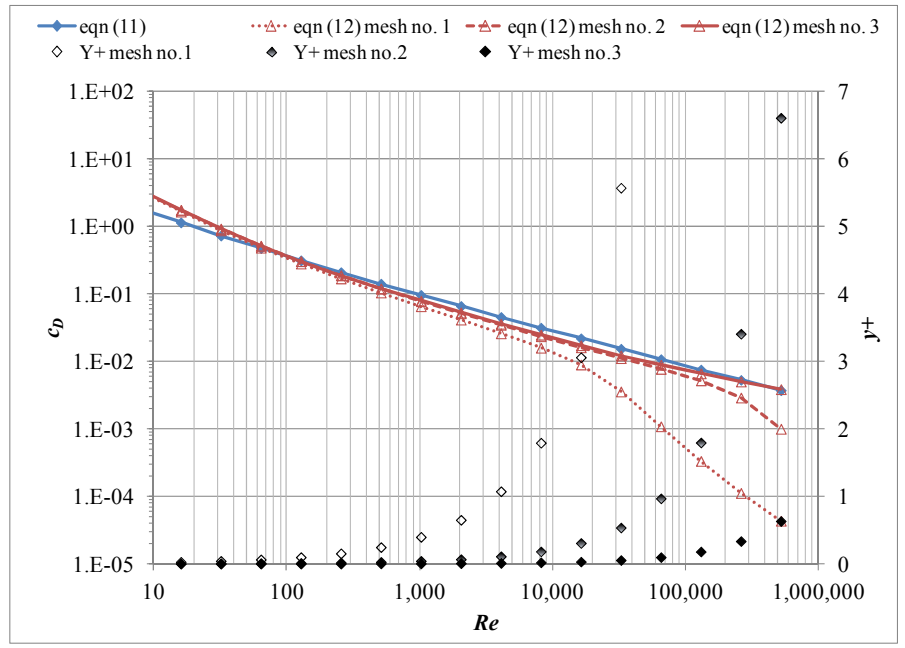

Figure 5: Drag coefficient for the semi-infinite flat plate.

\subsection{Internal flow: concentric rotating cylinders}

In a further study, the flow between two concentric rotating cylinders has been investigated as an example for an internal flow (corresponding to the local flow between the two cylinders for our canonical configuration at $\beta=0)$. The ratio between the outer and inner tangential velocity is defined as $\lambda=\omega_{1} r_{l} /\left(\omega_{0} r_{0}\right)$. The dimensionless radial distance is $R=r / r_{0}$. Fig. 6 shows the numerically calculated velocity profile compared to the analytical solutions from Mahmud and Fraser [14] for a varying $\lambda$, with $U=u /\left(\omega_{0} r_{0}\right)$.

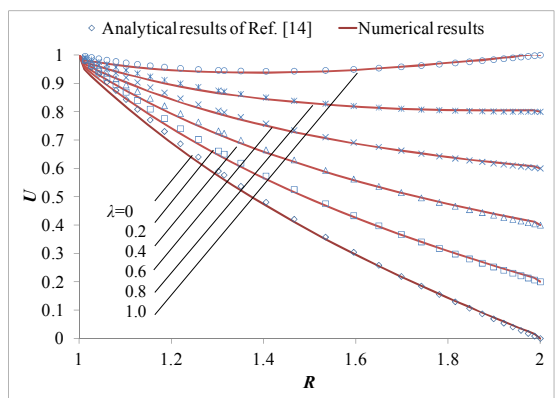

Figure 6: Effect of $\lambda$ on $U$ for $\beta=0^{\circ}$ $\Pi=2$ and $B r=0$.

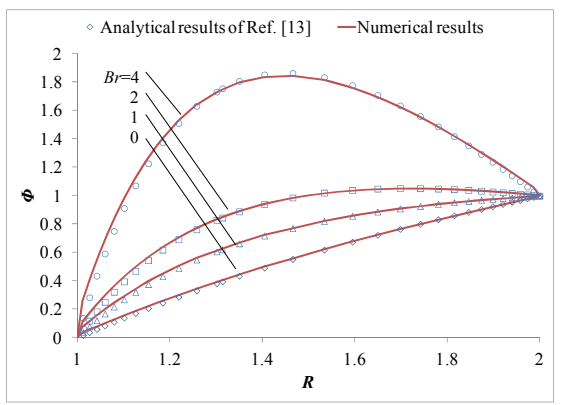

Figure 7: Effect of $B r$ on $\Phi$ for $\beta=0^{\circ}$, $\Pi=2$ and $\lambda=0$.

The numerical solutions are in excellent agreement with the analytical solution for the cases with $0 \leq \lambda \leq 1$, when the tangential velocity of the inner cylinder is constant and the outer tangential velocity is changed in magnitude. 
Table 3 shows the $y^{+}$values employed for each study. As appears from the table, the $y^{+}$values are much smaller than 1 .

Table 3: Y+ values at the inner and outer cylinder wall and Reynolds numbers in the gap for different values of $\lambda$.

\begin{tabular}{|l|c|c|c|c|c|c|}
\hline & $\lambda=0$ & $\lambda=0.2$ & $\lambda=0.4$ & $\lambda=0.6$ & $\lambda=0.8$ & $\lambda=1.0$ \\
\hline$y^{+}$inner cylinder & 0.088 & 0.084 & 0.079 & 0.074 & 0.068 & 0.062 \\
\hline$y^{+}$outer cylinder & 0.034 & 0.042 & 0.040 & 0.037 & 0.034 & 0.031 \\
\hline$R e$ in gap & 16.4 & 20.2 & 23.9 & 27.7 & 31.5 & 35.3 \\
\hline
\end{tabular}

Fig. 7 shows the numerical solutions for the dimensionless temperature profile $\Phi$ in comparison to the analytical solution from Mirzazadeh et al. [13]. Both cylinders are associated with an isothermal boundary condition. The results show, that the analytical solutions match almost perfectly with the numerical results. To vary $\mathrm{Br}$ for the temperature profiles, the dynamic viscosity $\mu$ was changed to $1.83 \times 10^{-2} \mathrm{~kg} \mathrm{~m}^{-1} \mathrm{~s}^{-1}$ for this study. Because of $\lambda=0, y^{+}$values were calculated for the inner cylinder, only (table 4).

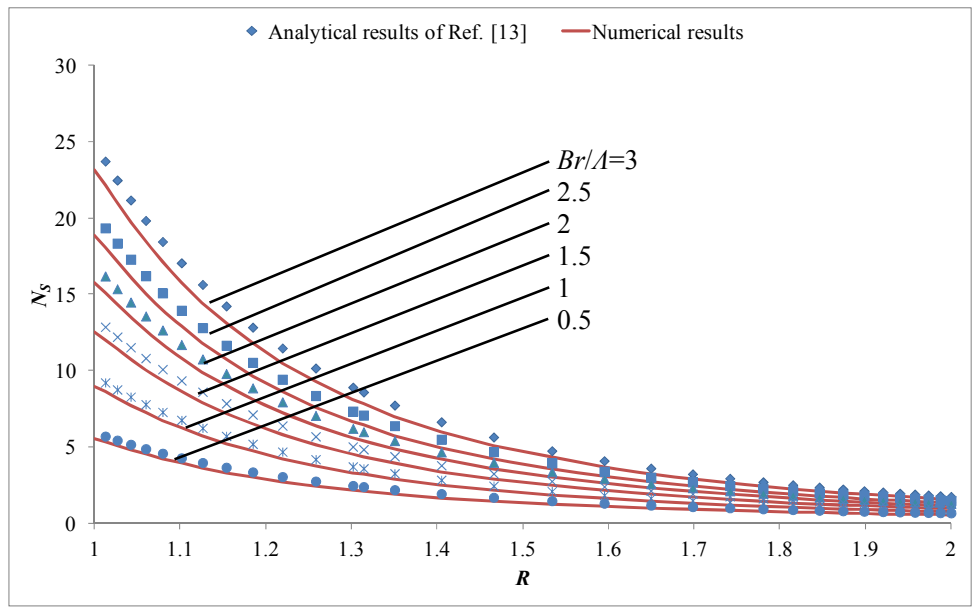

Figure 8: $\quad$ Effect of $B r / \Lambda$ on $N_{S}$ for $\beta=0^{\circ}, \Pi=2$ and $\lambda=0$.

Table 4: $Y^{+}$values at the inner cylinder for different values of $B r$.

\begin{tabular}{|l|c|c|c|c|}
\hline & $B r=0$ & $B r=1$ & $B r=2$ & $B r=4$ \\
\hline$y^{+}$inner cylinder & 0.003 & 0.01 & 0.012 & 0.016 \\
\hline$R e$ in gap & 0.01 & 0.2 & 0.3 & 0.5 \\
\hline
\end{tabular}

The entropy generation $\left(S_{g e n}^{\prime \prime \prime}\right)$ can be obtained by (see Bejan [3]):

$$
S_{g e n}^{\prime \prime \prime}=S_{C}^{\prime \prime \prime}+S_{F}^{\prime \prime \prime}=\frac{k}{T^{2}}(|\nabla T|)^{2}+\frac{\mu}{T}\left(\frac{\partial U_{i}}{\partial x_{j}}+\frac{\partial U_{j}}{\partial x_{i}}\right) \frac{\partial U_{i}}{\partial x_{j}} .
$$


The associated dimensionless form $N_{S}$ is equal to the ratio of $S_{g e n}^{\prime \prime \prime}$ to a characteristic entropy generation rate $\left(S_{G, C}\right)$. These are for both thermal boundary conditions:

$$
S_{G, C}=\left[\frac{q^{2}}{k T_{0}^{2}}\right]_{\text {Isoflux }} \quad S_{G, C}=\left[\frac{k(\Delta T)^{2}}{r_{0}^{2} T_{0}^{2}}\right]_{\text {Isothermal }} .
$$

Fig. 8 shows the dimensionless entropy generation number $N_{S}$ for a varying $B r / \Lambda$ ratio, compared to the analytical results from Mirzazadeh et al [13]. The highest relative derivation between theory and numeric is below $8 \%$ and is found for $B r / \Lambda=3$. Concerning the $y^{+}$and $R e$ values, the results in table 3 can be used.

As a whole, the validation of our numerical approach is successful. For most tested configurations and parameters, the numerical results are identical or nearly identical to the analytical prediction. Small discrepancies, below $8 \%$ are only observed for the internal flow between two concentric cylinders. An explanation for these larger differences could not be found yet. Value of $y^{+}$well below 1 are needed for an accurate analysis of flow, heat transfer, and associated entropy generation.

\section{Results and discussion}

Applying now the developed approach for a first study, the design parameters for our canonical configuration were set to $\beta=30^{\circ}$ and $\Pi=2$ with an adiabatic sleeve. Fig. 9 shows the resulting Nusselt number for varying angular velocities $\omega_{0}(\Gamma)$ and inlet velocities $u_{\infty}(R e)$.

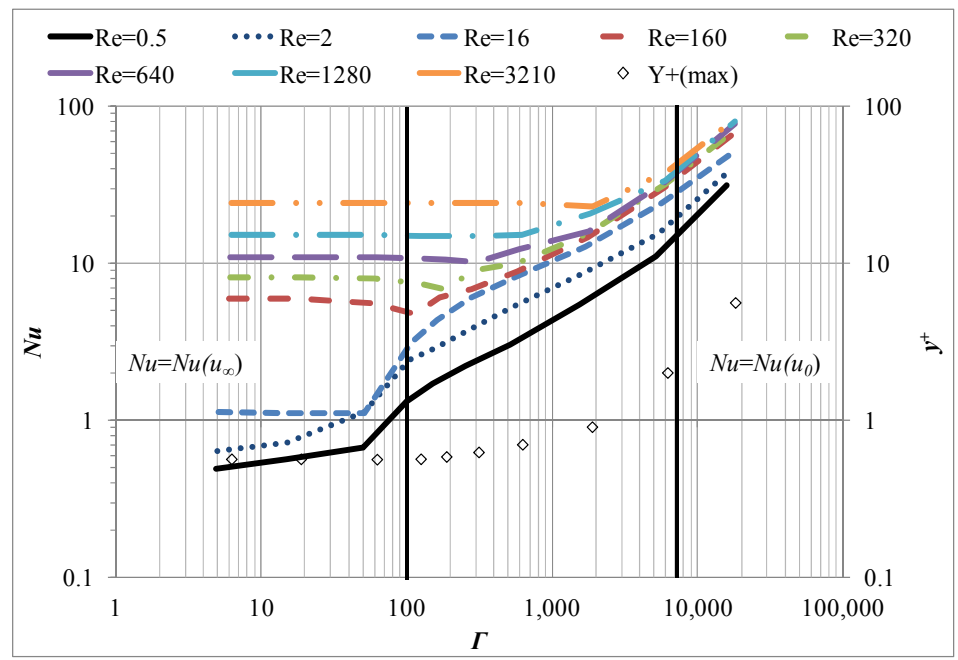

Figure 9: Effect of design parameters on $\mathrm{Nu}$ for $\beta=30^{\circ}, \Pi=2$ and $\mathrm{Br}=0$. 
When increasing $\Gamma$ the effect of the tangential speed of the cylinder $\left(u_{0}=\omega_{0} r_{0}\right)$ overweights the inlet velocity $u_{\infty}$. In the range $1 \leq \Gamma \leq 6000$ the Reynolds number is the dominating effect for $N u$. For the range $\Gamma>6000$ the heat and mass transfer mainly depend on the angular velocity $\omega_{0}$. The $y^{+}$values used in the simulation increase linearly with the magnitude of $N u$, so that the expected accuracy is lower in the right part of the diagram.

\section{Conclusions}

The first and second laws of thermodynamics have been used in combination with numerical simulations to investigate different configurations. A canonical configuration has been proposed based on fundamental geometries used for aerodynamic studies. This canonical configuration allows studying different transport phenomena correlated to different types of dynamic, heat transfer and design parameters. Numerical studies for external and internal flows were done to check grid-independence and for the purpose of code validation. Based on these first results, deriving suitable indicators based on entropy generation in the canonical configuration appears to be possible. As a first application example, the Nusselt number was calculated for $\beta=30^{\circ}, \Pi=2$ and $B r=0$. For the defined boundary conditions the transition area for $N u$ is between $100 \leq \Gamma \leq 6000$. At values of $\Gamma \leq 100$ the inlet velocity $u_{\infty}$ is the dominating effect. At higher values of $\Gamma$ the influence of the tangential velocity $u_{0}$ overweights $u_{\infty}$. Further studies will systematically vary the corresponding parameters in order to derive corresponding laws.

\section{References}

[1] Thévenin, D., Janiga, G., Optimization and Computational Fluid Dynamics, Springer-Verlag: Berlin and Heidelberg, 2008.

[2] VDI-GVC (ed.), VDI Heat Atlas, Springer-Verlag: Berlin and Heidelberg, 2010.

[3] Bejan, A., Entropy Generation Minimization, CRC Press: New York, 1995.

[4] Fowler, A.J. \& Bejan, A., Correlation of optimal sizes of bodies with external forced convection heat transfer. International Communications in Heat and Mass Transfer, 21(1), pp. 17-27, 1994.

[5] Herwig, H. \& Schmandt, B., Drag with external and pressure drop with internal flows: a new and unifying at losses in the flow field based on the second law of thermodynamics, Fluid Dynamics Research, 45(5), 2013.

[6] Wenterodt, T., Herwig, H., The Entropic Potential Concept: a New Way to Look at Energy Transfer Operations. Entropy, 16(4), pp. 2071-2084, 2014.

[7] Herwig, H., Gloss, D., \& Wenterodt, T., Flow in Channels With Rough Walls_-Old and New Concepts. Heat Transfer Engineering, 31(8), pp. 658-665, 2010. 
[8] Ko, T.H., \& Tink, K., Entropy generation and optimal analysis for laminar forced convection in curved rectangular ducts: A numerical study, International Journal of Thermal Sciences, 45, pp. 138-150, 2006.

[9] Ko, T.-H., A numerical study on entropy generation and optimization for laminar forced convection in rectangular curved duct with longitudinal ribs, International Journal of Thermal Sciences, 45, pp. 1113-1125, 2006.

[10] Şahin, A.Z., Irreversibilities in various duct geometries with constant wall heat flux and laminar flow. Energy, 23(6), pp. 465-473, 1998.

[11] Aziz, A., Entropy generation in pressure gradient assisted Couette flow with different thermal boundary conditions. Entropy, 8(2), pp. 50-62, 2006.

[12] Bejan, A., Second-Law Analysis in Heat Transfer and Thermal Design. Advances in Heat Transfer, 15, pp. 1-58, 1982.

[13] Mirzazadeh, M., Shafaei, A., \& Rashidi, F., Entropy analysis for nonlinear viscoelastic fluid in concentric rotating cylinders. International Journal of Thermal Sciences, 47(12), pp. 1701-1711, 2008.

[14] Mahmud, S. \& Fraser, R.A., Analysis of entropy generation inside concentric cylindrical annuli with relative rotation. International Journal of Thermal Sciences, 42(5), pp. 513-521, 2003.

[15] Mahian, O., Oztop, H., Pop, I., Mahmud, S., \& Wongwises, S., Entropy generation between two vertical cylinders in the presence of MHD flow subjected to constant wall temperature. International Communications in Heat and Mass Transfer, 44, pp. 87-92, 2013.

[16] Mahian, O., Mahmud, S. \& Heris, S.Z., Analysis of entropy generation between co-rotating cylinders using nanofluids. Energy, 44, pp. 438-446, 2012.

[17] Mahian, O., Mahmud, S. \& Pop, I., Analysis of first and second laws of thermodynamics between two isothermal cylinders with relative rotation in the presence of MHD flow. International Journal of Heat and Mass Transfer, 55(17-18), pp. 4808-4816, 2012.

[18] Yilbas, B. S., Entropy analysis of concentric annuli with rotating outer cylinder, Exergy. 1(1), pp. 60-66, 2001.

[19] Abu-Hijleh, B.A.K. \& Heilen, W.N., Entropy generation due to laminar natural convection over a heated rotating cylinder. International Journal of Heat and Mass Transfer, 42(22), pp. 4225-4233, 1999.

[20] Karabelas, S.J., Koumroglou, B.C., Argyropoulos, C.D. and Markatos, N.C., High Reynolds number turbulent flow past a rotating cylinder. Applied Mathematical Modelling, 36(1), pp. 379-398, 2012.

[21] Zhang, X., Chen, J., Kang, W., Wu, J., \& Yuan, T., Heat transfer coefficient calculation for analysis of ITER shield block using CFX and ANSYS, Fusion Engineering and Design, 86; pp. 312-317, 2011.

[22] ANSYS, Inc., ANSYS CFX-Solver Modeling Guide, Release 15.0, 2013. 